\title{
Circumnuclear stellar and gaseous disks in bulge-dominated disk galaxies
}

\author{
Olga K. Sil'chenko ${ }^{1}$ \\ ${ }^{1}$ Sternberg Astronomical Institute, Moscow State University, University av. 13, Moscow \\ 119992, Russia email: olga@sai.msu.su
}

\begin{abstract}
By means of 2D spectroscopy we have found a lot of compact inner stellar and gaseous disks in morphologically normal galaxies. The evolution and spatial orientation of these inner structures are often decoupled from the evolution and spatial orientation of the large-scale galactic disks and bulges. We argue that this finding requires substantial change of common approaches to investigations of the central parts of galaxies, including central black hole studies.
\end{abstract}

For the last 15 years we undertook study of the central parts of nearby early-type disk galaxies with the Multi-Pupil Spectrograph of the 6-m telescope of the Special Astrophysical Observatory of the Russian Academy of Sciences (for the description of 3 variants of the MPFS - see Afanasiev et al. 1990 and http://www.sao.ru/hq/lsfvo/devices/mpfs). For several galaxies we have also supplemented our data with analogous integral-field observations made by the SAURON team (Bacon et al. 2001, de Zeeuw et al. 2002); the data were taken from the ING Archive of the UK Astronomy Data Center. We have found a lot of chemically and kinematically decoupled cores which are mostly compact circumnuclear disks (for the kinematical illustration of this fact see also Emsellem et al. 2004). The stellar populations of these disks are on average younger than the stars of their surrounding bulges; and their planes are often inclined to the main symmetry planes of the outer large-scale disks. We are able to prove that the observed substructures are axisymmetric disks and not nuclear bars, because we have $2 \mathrm{D}$ velocity fields where the kinematical major axes are aligned with the major axes of the central isophotes.

The circumnuclear GASEOUS disks are often decoupled from the circumnuclear stellar disks and are even stronger inclined to the outer disk planes; in fact, we have found a considerable 'population' of inner polar rings. Inner gas polar rings are found as in spiral galaxies with the massive outer gas disks co-planar to the main symmetry planes (e.g. NGC 2841, Sil'chenko et al. 1997, and NGC 7217, Sil'chenko \& Afanasiev 2000) as well in many lenticular galaxies (Fig. 1). The latter are HI-rich lenticulars, and the orientations of the outer gas rings/disks appear to be decoupled from the inner polar rings. We think that the polar orientation of the inner gas rings relates in some way to bars or other triaxial structures in the galaxies under consideration.

These observational findings have important implications for approaches widely used in the various investigations concerning the central parts of normal galaxies. Namely,

(a) If one sees an elongated photometric structure in the center of a galaxy, which is not aligned with the outer line of nodes, it cannot be unambiguously treated as a nuclear bar unless you obtain a 2D velocity field and find a characteristic isovelocity turn; a combination of photometric and kinematical analyses is quite necessary to make a firm conclusion!

(b) If one sees decoupled stellar and gaseous subsystems in the center of a galaxy, especially, if an inner polar gas ring is present, it does not mean that one sees a product of a minor merger; the galaxy may possess a large-scale thin gas disk with quite normal 

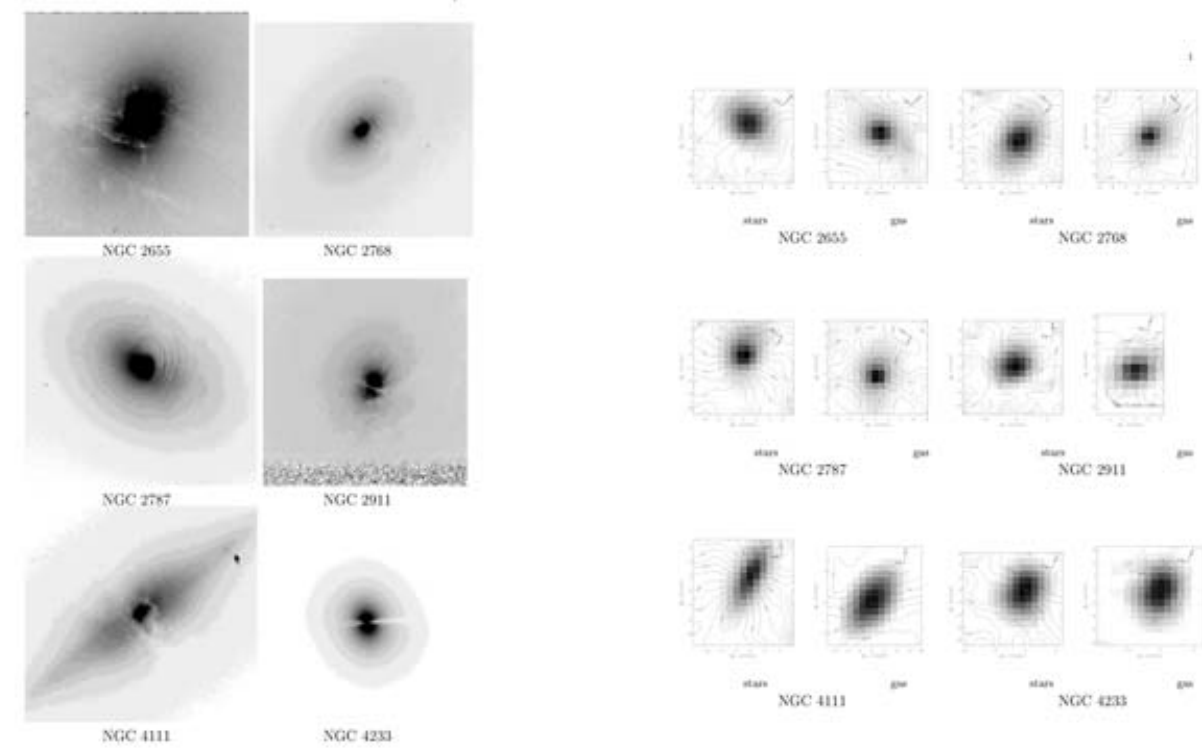

Figure 1. Some lenticular galaxies with the inner polar rings. $(a)$ The HST/WFPC2 view of the inner dust lanes $(b)$ The stellar (left) and ionized-gas (right) velocity fields obtained with the MPFS.

rotation and geometry. Some intrinsic mechanisms, a triaxial potential first of all, may put the inner gas into the polar orbits.

(c) A common approach when a dynamical model assuming spherical (isotropic) stellar distribution and constant stellar mass-to-light ratio along the radius is applied to observational data to determine a mass of the central black hole is quite wrong. Many galaxies possess a compact inner stellar disk with mass comparable to the mass of the black hole; it means that one must admit a two-component, very anisotropic stellar distribution and strongly variable $\mathrm{M} / \mathrm{L}$ because the inner disks and their surrounding bulges have different mean stellar ages and metallicities.

\section{Acknowledgements}

I thank my collaborators from the SAO RAS V. L. Afanasiev, A. N. Burenkov, A. V. Moiseev, and V. V. Vlasyuk. The study of young nuclei in lenticular galaxies has been supported by the Russian Foundation for Basic Research (grant 01-02-16167) and the study of the central parts of galaxies - by the Federal Scientific-Technical Program contract of the Science Ministry of Russia no.40.022.1.1.1101.

\section{References}

Afanasiev, V. L., Vlasyuk, V. V., Dodonov, S. N., \& Sil'chenko, O. K. 1990, Preprint of the SAO RAS, N54

Bacon, R., et al. 2001, MNRAS, 326, 23

Emsellem, E. et al. 2004, MNRAS, in press, (astro-ph/0404034)

Sil'chenko, O. K., Vlasyuk, V. V., \& Burenkov, A. N. 1997, A\&A, 326, 941

Sil'chenko, O. K., \& Afanasiev, V. L. 2000, A\&A, 364, 479

de Zeeuw, P. T., et al. 2002, MNRAS, 329, 513 